\title{
Detectors for leptonic CP violation at the Neutrino Factory
}

\section{A. Laing*t}

School of Physics \& Astronomy, University of Glasgow, Glasgow, UK.

E-mail: a.laing@physics.gla.ac.uk

The Neutrino Factory is the most powerful of the proposed facilities to search for CP violation in the lepton sector via neutrino oscillations. It delivers a well known beam of electron neutrinos and muon-antineutrinos from positive muon decay (electron-antineutrinos and muon neutrinos from negative muon decay) produced in the straight sections of the storage rings in which the muons are confined at an energy of $25 \mathrm{GeV}$. Studies carried out in the framework of the International Design Study for the Neutrino Factory (the IDS-NF) show that the sensitivity to the CP-violating phase, the mass hierarchy, and the last unknown mixing angle $\theta_{13}$ is maximised with far detectors able to detect with low background the sub-leading $v_{e}$ to $v_{\mu}$ oscillations. The IDS-NF baseline configuration calls for two detectors, one situated between $3000 \mathrm{~km}$ and $5000 \mathrm{~km}$ and another at the magic baseline of around $7500 \mathrm{~km}$. Several technologies are being discussed for these magnetised detectors: iron calorimeters; giant liquid argon TPCs; and totally active scintillating detectors. The IDS-NF baseline option - as a compromise between feasibility, cost, mass, and performance - is a 100 Kton magnetised iron sampling calorimeter at the intermediate baseline, similar to the existing MINOS detector but with 20 times more mass and improved performance, and a 50 Kton detector at the magic baseline. The other far-detector options, which have better granularity and offer both a lower energy threshold and an improved energy resolution, would complete the scientific return of the facility by detecting additional oscillation channels or unexpected effects and carry out a rich non-accelerator based neutrino programme. All options still require significant R\&D. A near detector of much smaller mass for precise measurement of neutrino flux and neutrino cross-sections will be situated close to the end of the muon storage ring straight section(s). The various detector options will be discussed.

35th International Conference of High Energy Physics - ICHEP2010,

July 22-28, 2010

Paris France

\footnotetext{
*Speaker.

${ }^{\dagger}$ on behalf of the IDS-NF collaboration
} 


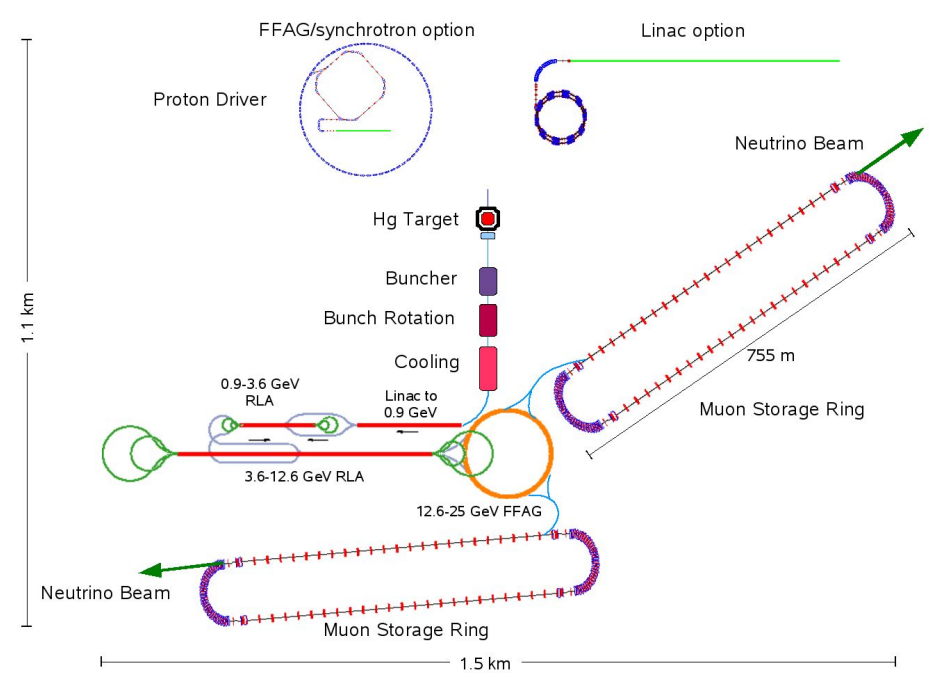

Figure 1: Schematic of the IDS-NF baseline for the Neutrino Factory accelerator complex.

\section{The Neutrino Factory and leptonic CP violation}

The Neutrino Factory is an accelerator facility creating an intense multi-flavour beam of neutrinos from the decay of muons which is currently being designed under the auspices of the IDSNF [1] and Euro- $v$ [2] collaborations. The primary physics goal of such a facility will be to measure the CP-violating phase $\delta_{C P}$ through the observation of sub-dominant appearance channels. A nonzero value for this quantity could have ramifications for the understanding of the matter antimatter asymmetry observed in the Universe.

In order to achieve this goal, it is envisioned that the facility (current design illustrated in Fig. 1) would have two muon storage rings, one with a straight section pointing towards a detector placed at $\sim 3000-5000 \mathrm{~km}$ and another pointing at a detector placed at $\sim 7500 \mathrm{~km}$. The combination of neutrino and antineutrino appearance signals taken at both of these detectors and cross-section and flux spectrum measurements taken at one or more detectors within $1 \mathrm{~km}$ of the facility was shown in [3] to be the experiment with the greatest coverage of the parameter space. The design of the detectors and analyses must include sufficient magnetic field to separate the signal interactions from those of the un-oscillated neutrinos as well as suppressing any background from the misidentification of non-oscillation neutrinos to at least the $10^{-3}$ level.

\section{Near detector}

The near detector design is still preliminary but it is clear that any detector must include a high-granularity tracking calorimeter with the ability to measure neutrino cross-sections for both neutrinos and anti-neutrinos of both flavours as well as measure key backgrounds which will not be easily identifiable at the far detectors. Some combination of a silicon vertex detector, to measure the production of charmed mesons, the leptonic and semi-leptonic decays of which can mimic primary oscillation muons, with a scintillating fibre or bar tracker should be sufficient to carry out the primary tasks. This basic design could also be augmented in order to exploit possible new physics channels. 


\section{Far detectors}

A number of possible designs are being considered in the design studies. All will be required to have high mass and be able to suppress backgrounds efficiently while maintaining signal efficiencies allowing measurement of the signal down to at least $3 \mathrm{GeV}$, this level was shown in [3] to be the threshold at which sensitivity to $\delta_{C P}$ saturates.

\subsection{Liquid Argon TPC}

One possible technology for the far detector would be a large liquid argon TPC (LAr TPC). The detection power of this technology for neutrinos has been shown recently by the ArgoNeuT [4] experiment, where the high position and energy resolution showed that this technology has great potential for neutrino detection. However, a great deal of $R \& D$ work will be required to prove that high mass can be achieved and that magnetisation is possible. The LAGUNA project [5] studied large LAr TPCs in underground sites. A clear advantage of this technology would be access to the $v_{e}\left(\bar{v}_{e}\right)$ and perhaps even $v_{\tau}\left(\bar{v}_{\tau}\right)$ appearance channels.

\subsection{Totally Active Scintillator Detector}

The totally active scintillator detector (TASD) would be comprised entirely of extruded plastic scintillator. Initial studies of this technology in the Neutrino Factory context [6] assumed a magnetic field of $0.5 \mathrm{~T}$ generated using a magnetic cavern constructed using superconducting transmission lines (STL) and showed a low energy threshold for $v_{\mu}$ detection should be achievable. Some efficiency for $v_{e}$ detection could also be achieved. However, significant $\mathrm{R} \& \mathrm{D}$ is required to realise the magnetic cavern concept and hadronic background suppression could be challenging.

\subsection{Magnetised Iron Neutrino Detector}

The Magnetised Iron Neutrino Detector (MIND) was chosen as the baseline detector for the Neutrino Factory in the ISS study [7] due to the large mass which can be achieved and relative ease with which such a detector can be magnetised. While this technology could not be used to detect channels other than those involving $v_{\mu}\left(\bar{v}_{\mu}\right)$ the statistical power of this channel means that $\delta_{C P}$ can be measured accurately with this channel alone [3].

Development of simulation and analysis tools to optimise this technology is underway with the first application of pattern recognition and analysis presented in [8]. It is envisioned that the same framework will be used to compare and contrast the different technologies, particularly to compare MIND to TASD which can be considered as the limit of the MIND technology as the iron content tends to zero.

The most recent analysis of MIND using a Geant4 [9] simulation and neutrino events generated using Nuance [10] results in the efficiencies shown in Fig 2 and suppresses all expected backgrounds to at or below the $10^{-4}$ level [11]. This level of suppression should allow measurement of $\mathrm{CP}$ violation over most of the currently allowed parameter space. 

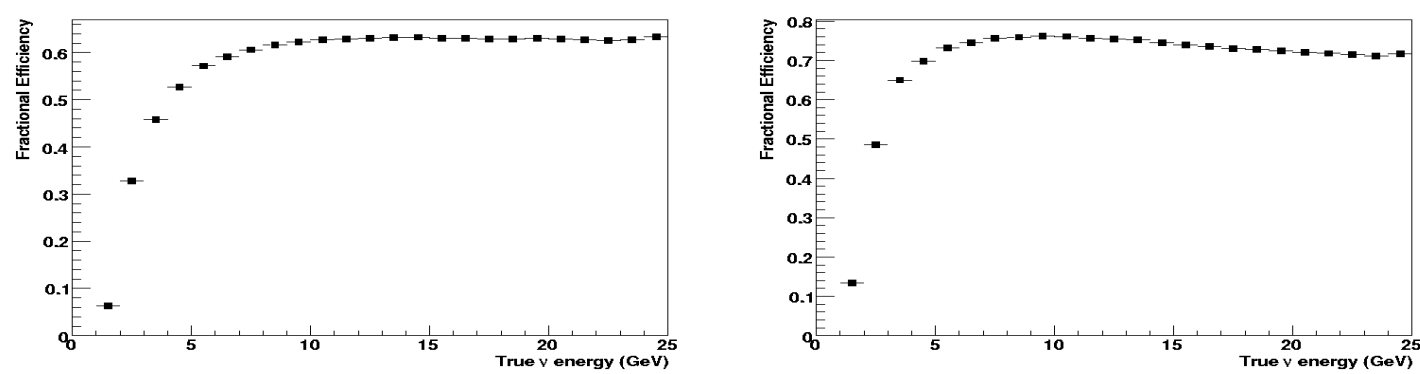

Figure 2: Signal efficiencies for the appearance of $v_{\mu}$ (left) and $\bar{v}_{\mu}$ (right) in the MIND detector. Details in [11]

\section{Conclusions}

The design and optimisation of Neutrino Factory detectors for the measurement of leptonic $\mathrm{CP}$ violation is underway. The latest results of simulation of the baseline detector design indicate that leptonic $\mathrm{CP}$ violation could be measured over most of the allowed parameter space.

\section{Acknowledgements}

I acknowledge the financial support of STFC and the European Community under the European Commission Framework Programme 7 Design Study: EUROnu, Project Number 212372.

\section{References}

[1] The International Design Study for the Neutrino Factory. URL: https://www.ids-nf.org/wiki/FrontPage.

[2] EUROnu: A High Intensity Neutrino Oscillation Facility in Europe. URL: http://www.euronu.org/.

[3] A. Bandyopadhyay et al. Physics at a future Neutrino Factory and super-beam facility. Rept. Prog. Phys., 72, 2009.

[4] Maddalena Antonello. ArgoNeuT and MicroBooNE: LAr-TPC's at Fermilab. AIP Conf. Proc., 1222:257-261, 2010.

[5] L. Oberauer. LAGUNA. Nucl. Phys. Proc. Suppl., 188:321-322, 2009.

[6] Alan D. Bross, Malcolm Ellis, Steve Geer, Olga Mena, and Silvia Pascoli. A Neutrino factory for both large and small $\theta_{13}$. Phys. Rev., D77:093012, 2008.

[7] T. Abe et al. Detectors and flux instrumentation for future neutrino facilities. JINST, 4:T05001, 2009.

[8] A. Cervera, A. Laing, J. Martín Albo, and F.J.P. Soler. Performance of the mind detector at a neutrino factory using realistic muon reconstruction. Nucl. Instrum. Meth. A, 624(3):601 - 614, 2010.

[9] Geant4 Physics Reference Manual. http://cern.ch/geant4/UserDocumentation/UsersGuides/ PhysicsReferenceManual/html/.

[10] D. Casper. The nuance neutrino physics simulation, and the future. Nucl. Phys. Proc. Suppl., 112:161-170, 2002.

[11] A Laing. Optimisation of detectors for the golden channel at a neutrino factory. PhD thesis, School of Physics \& Astronomy, University of Glasgow, 2010. 